\title{
Toll-like Receptors, LPS, and Dental Monomers
}

\section{G. Schmalz*, S. Krifka, and H. Schweikl}

Department of Operative Dentistry and Periodontology, University Medical Centre Regensburg, Franz-Josef-Strauss-Allee 11, 93042 Regensburg, Germany; *corresponding author, gottfried.schmalz@klinik .uni-regensburg.de

Adv Dent Res 23(3):302-306, 2011

\section{ABSTRACT}

Unreacted monomers released from dental resin-based composites at non-cytotoxic concentrations cause a depletion of glutathione and an increase of reactive oxygen species (ROS), leading to, e.g., DNA damage and apoptosis. ROS-sensitive MAP-kinases are activated by HEMA and TEGDMA. MAP-kinases are also involved in the bacteria-triggered cell responses of the innate immune system, e.g., after bacterial lipopolysaccharide (LPS) binding to the Toll-like receptor (TLR) 4 . Therefore, both bacteria and monomers imply environmental stress to pulp tissue, and they may influence the target cell reactions in a combined way. In macrophages, cell-surface antigens and cytokines were up-regulated after exposure to LPS, but TEGDMA caused a significant downregulation. Regulation was dependent on exposure time, indicating that LPS and TEGDMA act differently on MAP-kinases. Furthermore, the cell type played a decisive role. Inhibition of the immune response may result in a decrease in inflammatory symptoms and/or a reduced defense capacity against bacteria.

\section{CLINICAL BACKGROUND}

B esides damage to the dental pulp through, e.g., the caries process and cavity preparation trauma, further challenges for the dental pulp during restorative therapy derive from both dentin-contacting materials and bacteria. Resin-based composite materials (including adhesives) release unreacted monomers, especially the small and rather water-soluble molecules triethyleneglycol-dimethacrylate (TEGDMA) or 2-hydroxyethyl methacrylate (HEMA) (Hamid and Hume, 1997). Release is time-dependent, with about $90 \%$ of unreacted monomers being released during the first $24 \mathrm{hrs}$ after initial polymerization (Ferracane, 1995). Released monomers diffuse through dentin, and permeability (hydraulic conductance) of dentin is among other factors dependent on the diffusion distance (residual dentin thickness) in an inverse first-order polynomial way (Schmalz

\section{DOI: $10.1177 / 0022034511405391$}

(C) International \& American Associations for Dental Research et al., 2001). In deep cavities, the concentration of HEMA reaching the pulp would be in the range of $1.5-8 \mathrm{mmol} / \mathrm{L}$, and about 4 mmol/L for TEGDMA (Noda et al., 2002).

Inflammatory reactions (toxic reactions) are reported after the insertion of resin-based composites and adhesives in cavities very close to the pulp or when a (micro) perforation of the pulp had occurred. Consistently, the lack of new (osteo) dentin formation has been reported in humans when resin-based composites and the corresponding adhesives were in direct contact with the pulp, even when little inflammation was present (HörstedBindslev et al., 2003). Therefore, it is reasonable to assume from clinical observations that the released molecules (e.g., unreacted monomers) may interact with cell metabolism at concentrations that are not lethal to cells.

Another important challenge for the dental pulp in the context of restorative therapy is bacteria in the cavity under the restoration, either left behind or migrating between the cavity floor and resin material through a marginal gap. Despite improved bonding materials/techniques, marginal gaps have been reported both in vitro and in vivo, mainly at cavity margins located below the cemento-enamel junction (Hahn et al., 2008) and especially in technically challenging situations, e.g., in posterior teeth. Bacteria, especially their toxins (e.g., lipopolysaccharides for Gram-negative bacteria being present in deep caries lesions), may elicit pulp inflammation. It is further known that bacterial invasion inhibits dentin bridge formation after pulp capping (Rutherford and Gu, 2000). Therefore, both bacteria and monomers imply environmental stress to dental pulp tissue, and they may influence target cell reactions in a combined way.

\section{CELLULAR STRESS REACTIONS}

Monomer-related depletion of the non-enzymatic anti-oxidant glutathione $(\mathrm{GSH})$ and the increase of reactive oxygen species (ROS) are regarded as key steps in disturbing cellular homeostasis, with a reaction chain leading to DNA damage, activation of DNA repair mechanisms, and, eventually, apoptosis (Schweikl et al., 2006; Eckhardt et al., 2009b). This redox imbalance influences redox-sensitive signaling pathways of the cells which are responsible for cell survival and protective mechanisms (Matsuzawa and Ichijo, 2005; Schweikl et al., 2008). In this context, the activation of ROS-sensitive mitogen-activated protein kinases (MAP-kinases) by HEMA and TEGDMA has been demonstrated (Samuelsen et al., 2007; Krifka et al., 2010) (Fig. 1).

\section{Key Words}

resin monomer, innate immune system, stress response, MAPK, TEGMA, HEMA. 
MAP kinases, however, are also involved in the signal transduction of bacteria-triggered cell responses to the innate immune system. Lipopolysaccharides (LPS) bind to a special extracellular protein (LPB), then to cell-surface antigens CD14 and MD 2 , a complex that is recognized by the Toll-like receptor 4 (TLR4) in immune-competent cells like macrophages (Ulevitch, 2000; Miyake, 2004). By cell exposure to both TEGDMA and bacterial LPS, similar cellular signal transduction cascades are activated. Endpoints like the production of inflammation mediators and apoptosis, as well as the jointly activated MAP kinase system, will be discussed in more detail below (Fig. 1).

\section{INFLAMMATION MARKERS}

Inflammation as a host defense process is regulated by special signaling molecules like pro-inflammatory (e.g., IL-1, IL-6, TNF- $\alpha$ ) or by anti-inflammatory cytokines (e.g., IL-10), the latter preventing unlimited up-regulation of inflammation. Macrophages are target cells for cytokine production as part of the innate immune system, initiating and coordinating inflammatory processes (Janeway and Medzhitov, 2002). The regulation of these molecules has been studied repeatedly, and an influence of environmental stress factors on inflammation was shown.

Exposure to LPS $(25 \mu \mathrm{g} / \mathrm{mL}$, Escherichia coli $)$ on murine macrophages caused a large and time-related increase in the production of pro-inflammatory mediators IL- 6 or TNF- $\alpha$ or the anti-inflammatory mediator IL-10 in the cell culture supernatant. This was observed after 6 hrs of exposure, but was highest after $24 \mathrm{hrs}$ (e.g., an 800-fold increase for TNF- $\alpha$ ) (Eckhardt et al., 2009a). This was in agreement with reports from the literature (Hanada and Yoshimura, 2002) and data from Noda et al. (2003), who measured the TNF- $\alpha$ production of human THP-1 monocytes to $10 \mu \mathrm{g} / \mathrm{mL}$ LPS (E. coli) after a 24-hour exposure. Even a short exposure time (30 min) of $0.1 \mu \mathrm{g} / \mathrm{mL}$ LPS significantly enhanced the TNF- $\alpha$ concentration in murine macrophages, and this further increased with increasing exposure time. The secretion of IL- 6 and IL-10 started 2 hrs after exposure, but also increased until $24 \mathrm{hrs}$ of exposure (Krifka et al., 2010).

After exposure to $25 \mu \mathrm{g} / \mathrm{mL}$ LPS (E. coli), cell-surface antigens CD14, CD40, CD54, CD80, CD86, and MHC class I were time-dependently up-regulated, as shown by FACS analysis. For MHC-II, almost no effect was detected (Eckhardt et al., 2009a). These cell-surface antigens are involved in LPS/TLR4 recognition and the following signal transduction processes. CD14 is involved in the binding of LPS to TLR4, CD40 induces the expression of co-stimulatory molecules such as CD54 (ICAM1), CD80, and CD86, and MHC-I and MHC-II are antigenpresenting molecules (Roebuck and Finnegan, 1999; Tanaka et al., 2000; Fujii et al., 2004; Germain and Jenkins, 2004).

TEGDMA did not consistently up-regulate murine macrophage production of both IL- 6 and IL-10, but even decreased the release of IL- 6 (at 1 and 2 mM TEGDMA) and TNF- $\alpha(>1.0$ mM TEGDMA) after 6 hrs of exposure; IL-10 was not detectable (Eckhardt et al., 2009a). No cytokines could be measured after long exposure times (up to $48 \mathrm{hrs}$ ). This decreasing production of cytokines was not due to reduced cell viability, which was not affected by TEGDMA concentrations of up to $1 \mathrm{mM}$ after $6 \mathrm{hrs}$ of exposure (Eckhardt et al., 2009a). Noda et al. (2003) also
TEGDMA / HEMA

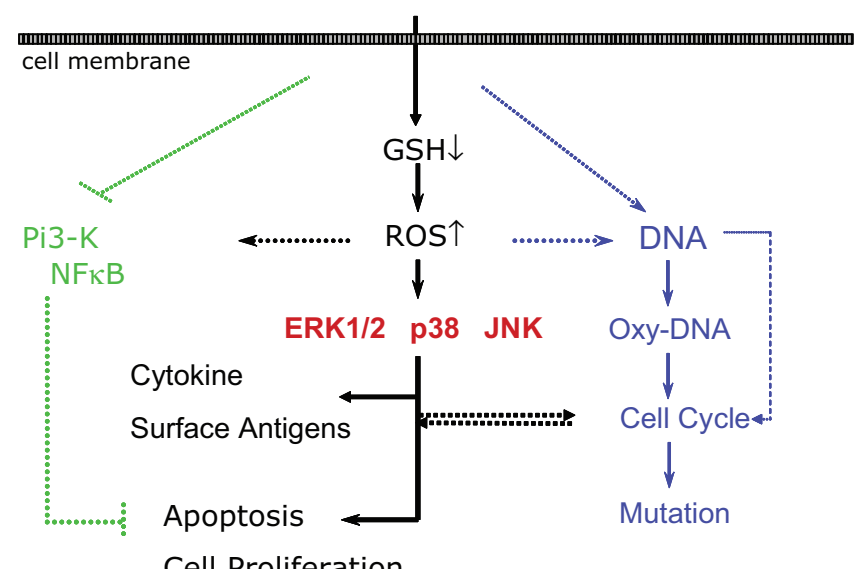

Figure 1. Cellular responses to dental monomers. In this model of cell responses exposed to dental monomers like TEGDMA and HEMA, the level of reactive oxygen species (ROS) is increased because of glutathione (GSH) depletion. The resulting oxidative stress leads to the activation of MAPK ERK $1 / 2$ (extracellular signal-regulated protein kinase), p38, and JNK (c-Jun N-terminal kinase), which play a central role in the regulation of cell responses as diverse as cytokine production, surface antigen expression, apoptosis, and cell proliferation. Dental monomers also cause DNA damage either directly or indirectly via ROS, which activates checkpoints regulating the progress through the cell cycle. Mutations in DNA sequences may occur in case of incomplete DNA repair.

reported no increased TNF- $\alpha$ release from human macrophages (THP1) after 2 wks of exposure to TEGDMA (up to $0.75 \mathrm{mM}$ ) and HEMA (up to1.2 mM). This is in contrast to data from in vitro experiments in a three-dimensional human epithelium model derived from a squamous cell carcinoma. Release of IL-6 was increased 5-fold after a 24-hour exposure to up to $20 \mathrm{mM}$ TEGDMA, which, in this model, did not cause cell death (Schmalz et al., 2000). TEGDMA had generally minor effects on the expression of cell-surface antigens. Some stimulation was observed, e.g., with MHC-I, CD 86, but only 2 mM TEGDMA significantly increased CD 54 (ICAM 1) over the level induced by $25 \mu \mathrm{g} / \mathrm{mL}$ LPS (Eckhardt et al., 2009a).

Exposure to both LPS $(25 \mu \mathrm{g} / \mathrm{mL}, E$. coli $)$ and TEGDMA in murine macrophages caused a significant down-regulation of IL-6, IL-10 ( $>0.125 \mathrm{mM}$ TEGDMA), and TNF- $\alpha(>0.25 \mathrm{mM}$ TEGDMA) compared with LPS stimulation only, as was measured in the cell culture supernatant. The pattern was similar for exposure periods from $6 \mathrm{hrs}$ to $48 \mathrm{hrs}$, but generally the amount of cytokines was greatest at 24-hour exposure (Eckhardt et al., 2009a). Under these experimental conditions, down-regulation responded to even small changes in TEGDMA concentrations that had not been observed in other cellular pathways (Spagnuolo et al., 2004; Samuelsen et al., 2007). This is in agreement with data from Noda et al. (2003) that TNF- $\alpha$ secretion was significantly suppressed by TEGDMA and HEMA in LPSpre-treated human macrophages. The down-regulation of LPSinduced cytokine production of murine macrophages after exposure to TEGDMA was also observed after short exposure times of, for instance, $30 \mathrm{~min}$ (Krifka et al., 2010). Also, the 
production of the cell-surface antigens CD 14, CD 40, CD 80, CD 86, and MHC class I was significantly down-regulated in LPS-stimulated murine macrophages after TEDMA $(>0.25$ $\mathrm{mM}$ ) exposure. In contrast, CD54 (= ICAM1) was up-regulated after 24 and $48 \mathrm{hrs}$ of exposure, and MHC-II antigens were also up-regulated at $24 \mathrm{hrs}$ of exposure to up to $0.5 \mathrm{mM}$ TEGDMA, then down-regulated, and at 6 and $48 \mathrm{hrs}$ of exposure time, no significant change was observed at all (Eckhardt et al., 2009a).

The reasons for the down-regulation of LPS-induced cytokine and cell-surface antigen production by TEGDMA are unknown. It was assumed that the TEGDMA-caused cellular redox imbalance might play a major role. The LPS-induced intracellular signaling cascade through the MyD88-dependent pathway generates ROS. Activation of further signaling may depend on the correct ROS concentration, which eventually was impaired by the TEGDMA-induced ROS increase (Eckhardt et al., 2009a).

\section{APOPTOSIS}

TEGDMA and HEMA have been shown to disturb cellular homeostasis, finally leading to apoptosis (Schweikl et al., 2006). The non-enzymatic anti-oxidant $\mathrm{N}$-acetylcysteine (NAC) protects cells from TEGDMA/HEMA-induced apoptosis (Schweikl et al., 2006; Paranjpe et al., 2009). LPS $(0.1 \mu \mathrm{g} / \mathrm{mL})$ increased the percentage of murine macrophages in late apoptosis after 15 and $30 \mathrm{~min}$ of exposure time, but no significant reduction of viable cells was observed. In contrast, human-pulp-derived cells (Galler et al., 2006) did not show any reaction to LPS under the given conditions (Krifka et al., 2010). In cell survival experiments, it was shown that LPS has a slight protective effect on TEGDMA-elicited cytotoxicity (Krifka et al., 2010). This is in agreement with data showing that LPS has an anti-apoptotic effect in different cell lines, including macrophages (Lombardo et al., 2007). TEGDMA, even at comparatively high concentrations of $3 \mathrm{mM}$, increased the amount of cells in apoptosis and late apoptosis only after 6 and more hours' exposure time, together with a significant decrease in viable cells. For $1 \mathrm{mM}$ TEGDMA, this was observed only after 24 hours' exposure time (Krifka et al., 2010). Human-pulp-derived cells did not generally show any increased level of apoptosis after exposure to TEGDMA until an exposure time of $2 \mathrm{hrs}$. Then a concentration-dependent increase of apoptosis and late apoptosis was observed. LPS and with TEGDMA-treated cell cultures showed the same pattern of apoptosis or necrosis as cultures exposed to TEGDMA alone. This was observed for murine macrophages and for human-pulpderived cells (Krifka et al., 2010).

\section{MAP KINASES}

The mitogen-activated protein kinases (MAP-kinases) ERK 1/2, $\mathrm{p} 38$, and $\mathrm{JNK}$ coordinate a variety of cellular responses: cell proliferation, induction of apoptosis, and the release of cytokines. P38 and JNK pathways are mainly activated by environmental stress including oxidative stress, but also by the bacterial lipopolysaccharide (LPS), and thus these kinases are also classified as stress-activated protein kinases (Matsuzawa and Ichijo,
2005). Differential activation of MAP-kinases p38, JNK, and ERK has also been described in the context of TEGDMA- and HEMA-induced apoptosis in different cell lines (Samuelsen et al., 2007; Spagnuolo et al., 2008; Eckhardt et al., 2009b).

For further elucidation of the reaction patterns of the single MAP-kinases after exposure to monomers and LPS, time kinetics of these reactions has been investigated. LPS $(0.1 \mu \mathrm{g} / \mathrm{mL}$, E. coli) exposure in murine macrophages caused an early activation with increased phosphorylation of ERK1/2 after 15/30 min and for $\mathrm{p} 38$ and JNK after 15 min of LPS exposure. After 1 and 2 hours' exposure, some activation was still observed for ERK1/2, but no p38 activation and no JNK were detected. At exposure times of 6 and $24 \mathrm{hrs}$, ERK1/2 showed a slight activation after $6 \mathrm{hrs}$, which further increased after $24 \mathrm{hrs}$; p38 was not activated after $6 \mathrm{hrs}$, but was significantly activated after $24 \mathrm{hrs}$, and JNK activation was not detected (Krifka et al., 2010).

In contrast, TEGDMA (3 mM) did not cause an early activation of any MAP-kinase in murine macrophages after 15 and 30 min, only a slight activation of p38 after 30 min. After 1 and 2 hours' exposure, ERK1/2 was inhibited by TEGDMA, and no p38 and JNK activation was detected. Still no activation of ERK1/2 was observed after $6 \mathrm{hrs}$, but a very strong activation was observed after 24 hours' exposure. P38 was slightly activated after 6 hrs and strongly activated after 24 hours' exposure to TEGDMA. JNK activation was not detected (Krifka et al., 2010). LPS/TEGDMA exposure in murine macrophages caused an early activation, with increased phosphorylation of ERK1/2 and JNK after 15 and $30 \mathrm{~min}$ and p38 after 15 minutes' exposure, just as had been seen with LPS alone. Similar results were detected after 1 and 2 hours' exposure to TEGDMA. In contrast, late activation (6 and 24 hours' exposure) caused an activation pattern very similar to that of TEGDMA exposure alone: ERK $1 / 2$ and p38 with a slight activation after 6 hrs, but a very strong activation after $24 \mathrm{hrs}$, and JNK activation was not detected (Krifka et al., 2010). In human transfected-pulpderived cells, LPS stimulation $(0.1 \mu \mathrm{g} / \mathrm{mL}, E$. coli $)$ under the given experimental conditions did not cause any activation of MAP kinases. Also, TEGDMA exposure did not activate ERK1/2 after 15 min until 2 hrs, but a considerable increase in activation occurred after 6 and especially after 24 hours' exposure. In parallel, no increase in activation for p38 and JNK up to 2 hours' exposure was detected, but a strong increase was observed for p38 after $24 \mathrm{hrs}$. LPS/TEGDMA exposure caused no ERK1/2 activation after $15 \mathrm{~min}$ until $2 \mathrm{hrs}$. LPS seemed to inhibit ERK1/2 increase after 6 and 24 hrs. No increase of p38 and JNK activation up to 2 hours' exposure was seen, but p38 activation increased after $24 \mathrm{hrs}$, although less than with TGDMA alone (Krifka et al., 2010).

\section{IMMEDIATE REACTION vs. SUSTAINED REACTION}

The reaction pattern of cells after exposure to LPS and TEGDMA (separately and together) is clearly characterized by a biphasic nature (Fig. 2). The immediate reaction (as early as 15 minutes' exposure) is characterized by an LPS-induced MAP-kinase activation in specialized target cells for LPS recognition (macrophages), with a consecutive up-regulation of inflammation 


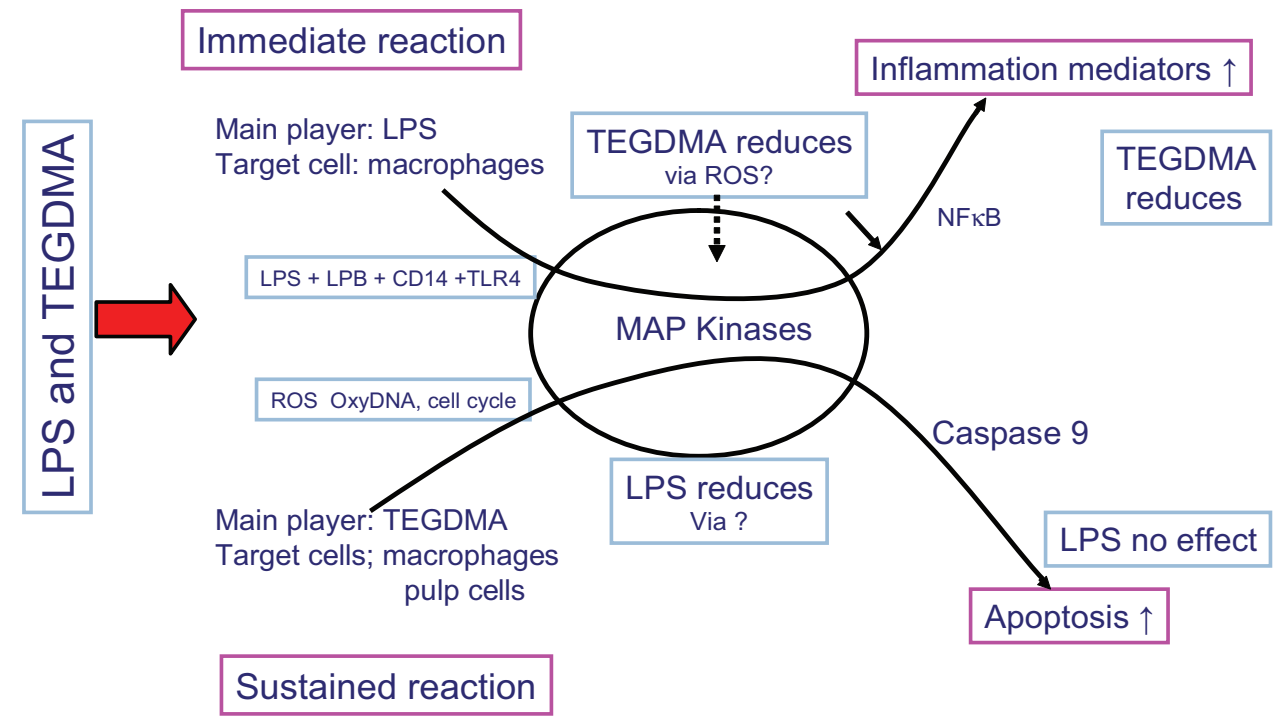

Figure 2. Stimulants of immediate and sustained responses in RAW264.7 mouse macrophages. Cells exposed to LPS (lipopolysaccharide) immediately activate MAP-kinases in target cells (macrophages), which then up-regulate inflammation mediators, including cytokines and related cellsurface antigens. This cell response is inhibited in the presence of dental monomers like TEGDMA, probably via ROS. In a sustained cell reaction, MAP-kinases are activated in response to TEGDMA exposure. The activation of MAP-kinases is associated with monomer-induced apoptosis. This cell reaction was detected in cells derived from various tissues, including macrophages and pulp cells.

markers (e.g., cytokines and related cell-surface antigens). TEGDMA significantly reduced this defense reaction of the innate immune system. The reason for this reduction remains unclear, although the monomer-induced redox imbalance may play an essential role. The immediate reaction after LPS/TEGDMA exposure is mainly determined by the LPS-mediated reaction.

A sustained reaction was observed after $6 \mathrm{hrs}$ and was even more pronounced after 24 hours' exposure: Again, MAP-kinases are increased, but this is time-related to the TEGDMA exposure, with a slight reduction of MAP-kinases by LPS, but with little or no effect on apoptosis. In this case, elevated activation of MAP-kinases led to apoptosis. Such a reaction apparently does not require specialized cells, but is more non-specific in nature. The sustained reaction after LPS/TEGDMA exposure is mainly determined by TEGDMA.

Data on cellular reactions reported here were mainly based on exposure to TEGDMA, which is a bifunctional methacrylate monomer. Similar results are expected for HEMA, albeit at higher concentrations, because of similar chemical properties but only one functional group per molecule (Yoshii, 1997; Schweikl et al., 2007).

\section{CONCLUSION}

Two ways by which monomers interfere with cell metabolism, based on a monomer-induced imbalance of the intracellular redox system, have been revealed: (1) DNA damage, with the consecutive activation of corresponding repair mechanisms and eventually apoptosis, has been shown (Eckhardt et al., 2009b). Bacterial LPS will not substantially interfere with these reactions, which are non-specific regarding cell type and which take time. (2)
Furthermore, inhibition of the initial and rapid defense reaction of the innate immune system (elicited through bacterial LPS recognition by target cells) is caused by monomers by down-regulating relevant MAP-kinases and the matching inflammation mediators. The physiological consequences of the monomer-induced DNA damage/repair and apoptosis have been related to the fact that these processes are highly energy-consuming (Shannan et al., 2006). Recently, we demonstrated that mineralization markers of human-pulp-derived cells and pulpal stem cells were down-regulated after exposure to non-lethal concentrations of TEGDMA. It was assumed that in cells involved in coping with cellular oxidative stress, the remaining available energy for cell differentiation might be insufficient (Galler et al., in press).

The physiological consequences of the TEGDMA-induced down-regulation of the innate immune system are unclear. The dental pulp accommodates $\mathrm{CD}^{+}$and $\mathrm{CD}^{+}$T-cells as well as macrophages and dendritic cells. Macrophages are among others involved in pulpal inflammation following caries (Jontell et al., 1998). It was also shown that LPS administration leads to inflammation in rat pulps (Bergenholtz et al., 1991). Therefore, it can be concluded that monomers will interfere with inflammatory processes in the dental pulp, and the innate immune response operates on a lower level of activity after exposure to these molecules. This may result in suppression or alleviation of inflammatory symptoms, with a potentially better basis for healing and regeneration. However, it was also reported that although too much TNF- $\alpha$ may lead to sepsis, too little may lead to a negative effect on bacterial clearance (Männel and Echtenacher, 2000; Hehlgans and Pfeffer, 2005). Therefore, the consequences of the monomer-induced reduced innate immune response remain to be elucidated. 


\section{ACKNOWLEDGMENT}

The authors received no financial support and declare no potential conflicts of interests with respect to the authorship and/or publication of this article.

\section{REFERENCES}

Bergenholtz G, Nagaoka S, Jontell M (1991). Class II antigen expressing cells in experimentally induced pulpitis. Int Endod $J$ 24:8-14

Eckhardt A, Harorli T, Limtanyakul J, Hiller KA, Bosl C, Bolay C, et al. (2009a) Inhibition of cytokines and surface antigen expression in LPSstimulated murine macrophages by triethylene glycol dimethacrylate. Biomaterials 30:1665-1674.

Eckhardt A, Gerstmayr N, Hiller KA, Bolay C, Waha C, Spagnuolo G, et al. (2009b). TEGDMA-induced oxidative DNA damage and activation of ATM and MAP kinases. Biomaterials 30:2006-2014.

Ferracane JL (1995). Current trends in dental composites. Crit Rev Oral Biol Med 6:302-318.

Fujii S, Liu K, Smith C, Bonito AJ, Steinman RM (2004). The linkage of innate to adaptive immunity via maturing dendritic cells in vivo requires CD40 ligation in addition to antigen presentation and CD80/86 costimulation. $J$ Exp Med 199:1607-1618.

Galler K, Schweikl H, Thonemann B, Schmalz G (2006). Human pulpderived cells immortalized with SV40. Eur J Oral Sci 114:138-146.

Galler KM, Schweikl H, Hiller KA, Cavender A, Bolay C, D'Souza R, et al. (2010). TEGDMA reduces mineralization in dental pulp cells. J Dent Res [Epub ahead of print, Dec 6, 2010] (in press).

Germain RN, Jenkins MK (2004). In vivo antigen presentation. Curr Opin Immunol 16:120-125.

Hahn P, Weyen G, Fischer P, Plogmann S, Hannig M (2008). Marginal and internal adaptation of composite restorations to dentin in vivo and in vitro. Am J Dent 21:356-360.

Hamid A, Hume WR (1997). A study of component release from resin pit and fissure sealants in vitro. Dent Mater 13:98-102.

Hanada T, Yoshimura A (2002). Regulation of cytokine signaling and inflammation. Cytokine Growth Factor Rev 13:413-421.

Hehlgans T, Pfeffer K (2005). The intriguing biology of the tumour necrosis factor/tumour necrosis factor receptor superfamily: players, rules and the games. Immunology 115:1-20

Hörsted-Bindslev P, Vilkinis V, Sidlauskas A (2003). Direct capping of human pulps with a dentin bonding system or with calcium hydroxide cement. Oral Surg Oral Med Oral Pathol Oral Radiol Endod 96:591-600.

Janeway CA Jr, Medzhitov R (2002). Innate immune recognition. Annu Rev Immunol 20:197-216.

Jontell M, Okiji T, Dahlgren U, Bergenholtz G (1998). Immune defense mechanisms of the dental pulp. Crit Rev Oral Biol Med 9:179-200.

Krifka S, Petzel C, Hiller KA, Frank EM, Bosl C, Spagnuolo G, et al. (2010). Resin monomer-induced differential activation of MAP kinases and apoptosis in mouse macrophages and human pulp cells. Biomaterials 31:2964-2975.

Lombardo E, Alvarez-Barrientos A, Maroto B, Boscá L, Knaus UG (2007). TLR4-mediated survival of macrophages is MyD88 dependent and requires TNF-alpha autocrine signalling. J Immunol 178:3731-3739.

Männel DN, Echtenacher B (2000). TNF in the inflammatory response. Chem Immunol 74:141-161.
Matsuzawa A, Ichijo H (2005). Stress-responsive protein kinases in redoxregulated apoptosis signaling. Antioxid Redox Signal 7:472-481.

Miyake K (2004). Innate recognition of lipopolysaccharide by Toll-like receptor 4-MD-2. Trends Microbiol 12:186-192.

Noda M, Wataha JC, Kaga M, Lockwood PE, Volkmann KR, Sano H (2002). Components of dentinal adhesives modulate heat shock protein 72 expression in heat-stressed THP-1 human monocytes at sublethal concentrations. J Dent Res 81:265-269.

Noda M, Wataha JC, Lockwood PE, Volkmann KR, Kaga M, Sano H (2003). Sublethal, 2-week exposures of dental material components alter TNF-alpha secretion of THP-1 monocytes. Dent Mater 19: 101-105.

Paranjpe A, Cacalano NA, Hume WR, Jewett A (2009). N-acetyl cysteine mediates protection from 2-hydroxyethyl methacrylate induced apoptosis via nuclear factor kappa B-dependent and independent pathways: potential involvement of JNK. Toxicol Sci 108:356-366.

Roebuck KA, Finnegan A (1999). Regulation of intercellular adhesion molecule-1 (CD54) gene expression. J Leukocyte Biol 66:876-888.

Rutherford RB, Gu K (2000). Treatment of inflamed ferret dental pulps with recombinant bone morphogenetic protein-7. Eur J Oral Sci 108: 202-206.

Samuelsen JT, Dahl JE, Karlsson S, Morisbak E, Becher R (2007). Apoptosis induced by the monomers HEMA and TEGDMA involves formation of ROS and differential activation of the MAP-kinases p38, JNK and ERK. Dent Mater 23:34-39.

Schmalz G, Schweikl H, Hiller KA (2000). Release of prostaglandin E2, IL-6 and IL-8 from human oral epithelial culture models after exposure to compounds of dental materials. Eur J Oral Sci 108:442-448.

Schmalz G, Hiller KA, Nunez LJ, Stoll J, Weis K (2001). Permeability characteristics of bovine and human dentin under different pretreatment conditions. J Endod 27:23-30.

Schweikl H, Spagnuolo G, Schmalz G (2006). Genetic and cellular toxicology of dental resin monomers. $J$ Dent Res 85:870-877.

Schweikl H, Hartmann A, Hiller KA, Spagnuolo G, Bolay C, Brockhoff G, et al. (2007). Inhibition of TEGDMA and HEMA-induced genotoxicity and cell cycle arrest by N-acetylcysteine. Dent Mater 23:688-895.

Schweikl H, Hiller KA, Eckhardt A, Bolay C, Spagnuolo G, Stempfl T, et al. (2008). Differential gene expression involved in oxidative stress response caused by triethylene glycol dimethacrylate. Biomaterials 29:1377-1387.

Shannan B, Seifert M, Boothman DA, Tilgen W, Reichrath J (2006). Clusterin and DNA repair: a new function in cancer for a key player in apoptosis and cell cycle control. J Mol Histol 37:183-188.

Spagnuolo G, Galler K, Schmalz G, Cosentino C, Rengo S, Schweikl H (2004). Inhibition of phosphatidylinositol 3-kinase amplifies TEGDMA-induced apoptosis in primary human pulp cells. $J$ Dent Res 83:703-707.

Spagnuolo G, D'Antò V, Valletta R, Strisciuglio C, Schmalz G, Schweikl H, et al. (2008). Effect of HEMA on human pulp cells survival pathways ERK and Akt. $J$ Endod 34:684-688.

Tanaka J, Asaka M, Imamura M (2000). T-cell co-signalling molecules in graft-versus-host disease. Ann Hematol 79:283-290.

Ulevitch RJ (2000). Molecular mechanisms of innate immunity. Immunol Res 21:49-54.

Yoshii E (1997). Cytotoxic effects of acrylates and methacrylates: relationships of monomer structures and cytotoxicity. J Biomed Mater Res 37:517-524. 